\title{
Turbulent transition by photon-correlation spectroscopy
}

\author{
P. Tong, W. I. Goldburg, and C. K. Chan \\ Department of Physics and Astronomy, University of Pittsburgh, Pittsburgh, Pennsylvania 15260 \\ A. Sirivat \\ Department of Mechanical Engineering, University of Pittsburgh, Pittsburgh, Pennsylvania 15261
}

(Received 18 May 1987)

The technique of photon-correlation spectroscopy was exploited to study turbulent pipe flow behind a grid. We measure the correlation function $g(t)$ of the light intensity scattered by small particles suspended in the turbulent fluid. The results imply that the probability distribution function for the small relative velocity fluctuations in the turbulent grid flow is Lorentzian-like. The statistical properties of the small velocity fluctuations over varying length scales possess a self-similar feature. This self-similarity was seen only when the Reynolds number becomes larger than a specific value $\mathcal{R}_{c}$. All the measurements suggest that the flow changes its character at this point.

\section{INTRODUCTION}

A quantity of fundamental interest in the theory of turbulence is the velocity difference $\mathbf{V}(R, t)$, that a pair of points in the turbulent fluid separated by a distance $R$ differ in velocity by an amount $\mathbf{V}(R, t)=\mathbf{v}(r(t))-\mathbf{v}(r(t)$ $+R)$, where $\mathrm{v}(r(t))$ is the local velocity of the fluid. The statistical property of the velocity fluctuations $\mathbf{V}(R, t)$ can be characterized by its moments, $\left\langle|\mathbf{V}(R, t)|^{n}\right\rangle$, or more generally, by its probability distribution function, $P(\mathrm{~V}(R))$. $^{1,2}$

The distribution function $P(V(R))$ is accessible by the technique of photon correlation spectroscopy ${ }^{3}$ (PCS), as was first demonstrated many years ago by Bourke et al. ${ }^{4}$ The photon correlation technique used here differs from the standard one of laser Doppler velocimetry (LDV), ${ }^{5,6}$ in that LDV measures the local velocity $v(r(t))$ whereas photon correlation spectroscopy senses the instantaneous velocity difference, $\mathbf{V}(R, t)$. With the PCS scheme, the scattering is produced by small seed particles in the fluid, which follow the local flow. The photodetector records the scattered light, which is the beating of Dopplershifted light scattered by pairs of flowing particles. The output of the detector is therefore modulated at a frequency equal to the difference in Doppler shifts of all particle pairs in the scattering volume. For each particle pair, separated by a distance $R$, this difference is

$$
\mathbf{q} \cdot[\mathbf{v}(r(t))-\mathbf{v}(r(t)+R)]=\mathbf{q} \cdot \mathbf{V}(R, t),
$$

where the scattering vector $\mathbf{q}$ has the amplitude

$$
q=(4 \pi n / \lambda) \sin (\theta / 2) .
$$

Here $\theta$ is the scattering angle, $n$ the refractive index of the fluid, and $\lambda$ is the wavelength of the incident light. With this so-called homodyne method, one records the intensity correlation function $g(t)=\left\langle I\left(t^{\prime}\right) I\left(t^{\prime}+t\right)\right\rangle$, where $I(t)$ is the intensity of scattered light, and the angular brackets represent an ensemble average over many realizations. The PCS technique can also be used to probe velocity gradients in laminar flows, ${ }^{7}$ in which case the ensemble average is not needed because the flow is deterministic.

It will be seen that when $\mathbf{V}(R, t)$ is a stochastic variable, as in turbulence, the light scattered by each pair of particles suspended in the turbulent fluid contributes a phase factor $\cos [\mathbf{q} \cdot \mathbf{V}(R) t]$ (due to frequency beating) to the intensity correlation function, $g(t)$, and $g(t)$ is an incoherent sum of these ensemble averaged (or time averaged) phase factors over all the particle pairs in the scattering volume. The ensemble average of the phase factor $\cos [\mathbf{q} \cdot \mathbf{V}(R) t]$ involves the velocity distribution function $P(V(R))$. When the distribution function $P(\mathbf{V}(R))$ has the scaling form $P(|\mathbf{V}(R)| / \bar{u}(R))$ discussed below, the ensemble average of the phase factor $\cos [\mathbf{q} \cdot \mathbf{V}(R) t]$ becomes the Fourier cosine transform of $P(V(R))$. Therefore the measurement of $g(t)$ yields a weighted integral of the Fourier cosine transform of $P(\mathbf{V}(R))$. (This weighting is required, because the detector is sensitive to all particle pairs in the scattering volume, and for small $R$, more pairs will be found in the scattering volume than that for larger $R$.) When the direction of the scattering vector $q$ is fixed, the onedimensional distribution function $P\left(V_{\mathrm{q}}(R)\right)$ can be measured, where $V_{\mathrm{q}}(R)$ is the component of $\mathrm{V}(R, t)$ along the scattering vector $\mathbf{q}$ (we drop the subscript $\mathbf{q}$ hereafter when no confusion arises). The PCS technique yields information about velocity fluctuations without introducing an invasive probe, such as a hot wire anemometer. ${ }^{8}$ Nor is it necessary to invoke Taylor's "frozen turbulence" assumption ${ }^{9}$ to interpret the measurements.

In theories of fully developed turbulence, ${ }^{10,11,2,12}$ the dynamic process of turbulence is considered as a cascade of turbulent kinetic energy from large scales to small scales. Energy fed into the turbulence goes primarily into the large eddies. The size of these eddies is determined by the boundary of the system and establishes the outer scale $L_{0}$ of turbulence. From these large eddies, smaller 
eddies are generated, and then still smaller ones. The process continues until the length scale is small enough for viscous dissipation to occur. The smallest length scale of the energy cascade is called the Kolmogorov dissipation length ${ }^{10} L_{d}$, which is equal to $\left(v^{3} / \varepsilon\right)^{1 / 4}$, where $v$ is the kinematic viscosity of the fluid, $\varepsilon$ is the local energy dissipation rate. According to the Kolmogorov theory, ${ }^{10}$ only two parameters, $R$ and $\varepsilon$, are relevant to the turbulence at scales in the inertial range, $L_{d}<R<<L_{0}$.

A question of fundamental interest in the theory of turbulence is how the statistical property of the relative velocity fluctuations $\mathbf{V}(R, t)$ varies with the length scale $R$, when $R$ is in the inertial range. It was shown that ${ }^{10,2}$ for fully developed turbulence, the velocity fluctuations $\mathbf{V}(R, t)$ are self-similar, i.e., the statistical properties of $\mathbf{V}(R, t)$ over varying length scales $R$ become identical under an appropriate scaling of velocities. This implies that $\left\langle|\mathbf{V}(R, t)|^{n}\right\rangle$ is proportional to a power of $R$. It is easy to show that ${ }^{1}$ this self-similarity follows if the distribution function $P(\mathbf{V}(R))$ is of the form $P(|\mathbf{V}(R)| / \bar{u}(R))$, where $\bar{u}(R)$ is a scaling velocity associated with length scale $R$. Theoretical models have been developed ${ }^{1,13,14}$ and have led to predictions of self-similarity of fully developed turbulence. Experiments at very large values of Reynolds number in air channels, ducts, and in the atmosphere confirm some of these predictions. ${ }^{15,16}$ In contrast, very little is known about "real" turbulence at moderate Reynolds numbers. We use the word "real" to distinguish the turbulence being studied here from the chaotic behavior of nonlinear systems with only a few degrees of freedom. The experiments described here are an investigation of the turbulent flow at intermediate Reynolds numbers.

We explore turbulence in the familiar geometry of pipe flow through a grid. ${ }^{17}$ The Reynolds number of this grid flow is defined as $R=\mathrm{MU} / v$, where $\mathrm{U}$ is the mean flow velocity at the center line of the pipe, $M$ the aperture size of the grid which generates turbulence, and $v$ is the kinematic viscosity $\left(v=0.01 \mathrm{~cm}^{2} / \mathrm{sec}\right.$ in water). Several notable features of the turbulent flow were found. When the Reynolds number becomes larger than a transition Reynolds number $R_{c}(\sim 460)$, the intensity correlation function $g(t)$ obeys a scaling form, $g(t)=g(x)$, where

$$
x=q \bar{u}(L) t\left(L_{0} / L\right)^{\gamma}
$$

Here $L$ is the size of the scattered beam viewed by the photodetector, and $\bar{u}(L)$ is the characteristic velocity associated with an eddy of size $R=L$. In the Kolmogorov theory ${ }^{18} \bar{u}(R)=(\varepsilon R)^{1 / 3}$. The largest scale of turbulence generated by the grid is denoted as $L_{0}$ which is proportional to the grid aperture size $M$. According to Eq. (1.1) the characteristic decay time of the decaying function $g(t)$ is of the order of $\left[q \bar{u}(L)\left(L_{0} / L\right)^{\gamma}\right]^{-1}$. Because the inverse decay time is proportional to $q$ rather than $q^{2}$, it is clear that the relaxation of the velocity fluctuations cannot be characterized by a turbulent diffusivity.

Both the functional form of $g(x)$ and its scaling argument $x$ provide information about statistical properties of $V(R, t)$. Our measurements of $g(t)$ suggest that the dis- tribution function $P(V(R))$ is of Lorentzian form for small values of $V(R, t)$ when $R \gtrsim R_{c}$. Equivalently the characteristic function [the Fourier transform of $P(V(R))$ ] decays exponentially. The exponent $\gamma$ in Eq. (1.1) shows a nontrivial $\mathcal{R}$ dependence, and reveals a transition character when $\mathcal{R}$ is near and above $\mathcal{R}_{c}$. By measuring how the characteristic decay time of $g(t)$ depends on $L$, we extract the $R$ dependence of $\zeta$ $(\zeta=1 / 3-\gamma)$. A plot of $\zeta$ versus $R$ exhibits a kink at $R_{c}$ $(\sim 460)$. When $R$ is near and above $R_{c}$, it was found that

$$
\zeta(\mathscr{R}) \sim\left\{\left[\mathcal{R}-\mathscr{R}_{c}\right] / \mathcal{R}_{c}\right\}^{\alpha},
$$

where $\alpha$ lies between 0.5 and 0.2 . When $\mathcal{R} \gtrsim 1400, \zeta$ has climbed to and saturated at a value close to $\frac{1}{3}$ (the Kolmogorov value). Our measurements of $g(t)$ indicate that in the range of $460 \leqslant R \approx 3600$, the concept of the selfsimilarity can still be applied to the velocity fluctuations even though the flow is far from being fully developed.

The next section of this paper contains the derivation of the correlation function $g(t)$ of the light intensity scattered by the particles in the turbulent fluid. Experimental details appears in Sec. III and the results are presented and analyzed in Sec. IV. Finally the work is summarized in Sec. $V$.

\section{THEORY}

The starting point is to consider the electric field scattered by $N$ identical particles suspended in a turbulent fluid. The diameter of the particles is taken to be small compared to the wavelength $\lambda$ of incident light. In the case where the polarization of the incident beam is perpendicular to the scattering plane, the scattered electric field $E(t)$ is a simple sum of the fields radiated by each of the particles in the scattering volume, and has the form ${ }^{4}$

$$
\begin{aligned}
E(t) & =\sum_{j=1}^{N} E_{s j}(t) \\
& =E_{0} \exp (-i \omega t) \sum_{j=1}^{N} \exp \left[-i \mathbf{q} \cdot \mathbf{r}_{j}(t)\right] .
\end{aligned}
$$

Here $\mathbf{r}_{j}(t)$ is the trajectory of the $j$ th particle inside the scattering volume, $\omega$ is the angular frequency of the incident light, and $E_{0}$ is a proportionality factor involving the distance from source to detector, relative refractive index of the particles, and the wavelength $\lambda$.

The correlation function of the light intensity scattered by the particles in the turbulent fluid is, by definition,

$$
g(t)=\frac{\left\langle E^{*}\left(t^{\prime}\right) E\left(t^{\prime}\right) E^{*}\left(t^{\prime}+t\right) E\left(t^{\prime}+t\right)\right\rangle}{\left|\left\langle E^{*}\left(t^{\prime}\right) E\left(t^{\prime}\right)\right\rangle\right|^{2}}=K / Q^{2},
$$

where the angle brackets represent the time average over $t^{\prime}$. Here

$$
\begin{aligned}
K=\left\langle\sum _ { k , j , m , n } ^ { N } \operatorname { e x p } \left\{i \mathbf { q } \cdot \left[\mathbf{r}_{j}\left(t^{\prime}\right)-\mathbf{r}_{k}\left(t^{\prime}\right)\right.\right.\right. \\
\left.\left.\left.+\mathbf{r}_{m}\left(t^{\prime}+t\right)-\mathbf{r}_{n}\left(t^{\prime}+t\right)\right]\right\}\right\rangle,
\end{aligned}
$$


and

$$
\begin{aligned}
Q & =\sum_{k, j=1}^{N}\left\langle\exp \left\{i \mathbf{q} \cdot\left[\mathbf{r}_{j}\left(t^{\prime}\right)-\mathbf{r}_{k}\left(t^{\prime}\right)\right]\right\}\right\rangle \\
& =N+\sum_{\substack{k, j=1 \\
k \neq j}}^{N}\left\langle\exp \left\{i \mathbf{q} \cdot\left[\mathbf{r}_{j}\left(t^{\prime}\right)-\mathbf{r}_{k}\left(t^{\prime}\right)\right]\right\}\right\rangle=N .
\end{aligned}
$$

The fluctuation terms $(k \neq j)$ do not survive a time or en- semble average if the scatterers are randomly distributed in the fluid.

Since the particles are assumed to follow the motion of the fluid, we have

$$
\mathbf{r}_{j}\left(t^{\prime}+t\right)=\mathbf{r}_{j}\left(t^{\prime}\right)+\int_{t^{\prime}}^{t^{\prime}+t} d t^{\prime \prime} \mathbf{v}\left(\mathbf{r}_{j}\left(t^{\prime \prime}\right)\right),
$$

where $\mathbf{v}\left(\mathbf{r}_{j}(t)\right)$ is the velocity field of the flow at position $\mathbf{r}_{j}$. Then Eq. (2.3) becomes

$$
K=\sum_{k, j, m, n=1}^{N}\left\langle\exp \left\{i \mathbf{q} \cdot\left[\mathbf{r}_{j}\left(t^{\prime}\right)-\mathbf{r}_{k}\left(t^{\prime}\right)+\mathbf{r}_{m}\left(t^{\prime}\right)-\mathbf{r}_{n}\left(t^{\prime}\right)\right]\right\} \exp \left\{i \mathbf{q} \cdot \int_{t^{\prime}}^{t^{\prime}+t} d t^{\prime \prime}\left[\mathbf{v}\left(\mathbf{r}_{m}\left(t^{\prime \prime}\right)\right)-\mathbf{v}\left(\mathbf{r}_{n}\left(t^{\prime \prime}\right)\right)\right]\right)\right\rangle
$$

With the same assumption as we made for Eq. (2.4), the fluctuation terms in Eq. (2.6) may be dropped, and the remaining terms satisfy the condition [see Eq. (2.4)],

$$
\mathbf{r}_{j}(t)-\mathbf{r}_{k}(t)+\mathbf{r}_{m}(t)-\mathbf{r}_{n}(t)=0,
$$

when $\mathbf{q} \neq 0$. The two possible choices to satisfy Eq. (2.7) are $j=k, m=n, j \neq m$ and $j=n, m=k, m \neq n$. On dropping the inconsequential contribution from the term $k=j=m=n$, Eq. (2.6) becomes

$$
\begin{aligned}
K & =N^{2}+\sum_{m \neq n}^{N}\left\langle\exp \left\{i \mathbf{q} \cdot \mathbf{V}\left(\mathbf{R}(m, n), t^{\prime}\right) t\right\}\right\rangle \\
& =N^{2}+2 \sum_{m>n}^{N}\left\langle\cos \left[\mathbf{q} \cdot \mathbf{V}\left(\mathbf{R}(m, n), t^{\prime}\right) t\right]\right\rangle,
\end{aligned}
$$

and

$g(t)=1+\left(2 / N^{2}\right) \sum_{m>n}^{N}\left\langle\cos \left[\mathbf{q} \cdot \mathbf{V}\left(\mathbf{R}(m, n), t^{\prime}\right) t\right]\right\rangle$.

Here $\mathbf{V}(\mathbf{R}(m, n), t)=\mathbf{v}\left(\mathbf{r}_{m}(t)\right)-\mathbf{v}\left(\mathbf{r}_{m}(t)+\mathbf{R}(m, n)\right)$, and $\mathbf{R}(m, n)=\mathbf{r}_{n}-\mathbf{r}_{m}$. The integral in Eq. (2.6) is replaced by $\mathbf{V}(\mathbf{R}(m, n), t) t$ because the shortest turbulent eddy turnover time, $t_{L_{d}}$, is much longer than the time $t$ of interest in our experiment.

When $N \gg 1$, the summation in Eq. (2.9) can be converted into an integration over the scattering volume. Therefore Eq. (2.9) becomes ${ }^{19}$

$$
g(t)=1+\int_{0}^{L} d R h(R)\left\langle\cos \left[\mathbf{q} \cdot \mathbf{V}\left(R, t^{\prime}\right) t\right]\right\rangle .
$$

Here the scattering volume is assumed to be quasi-onedimensional with length $L$, and $h(R)$ is the number fraction of particle pairs separated by distance $R$ in the scattering volume. When $N$ particles are evenly distributed in a one-dimensional scattering volume with length $L$, the fraction of particle pairs separated by $R$ is

$$
\begin{aligned}
h(R) & =(N-n) /[N(N-1) / 2] \\
& \simeq(2 / N)(1-n / N)=(2 / L)(1-R / L) .
\end{aligned}
$$

If we assume the ensemble average of the turbulent velocity is equivalent to its time average, Eq. (2.10) may be written in an alternative form

$$
\begin{aligned}
g(t)=1+\int_{0}^{L} d R h(R) \int_{-\infty}^{\infty} & d V_{q}(R) P\left(V_{\mathrm{q}}(R)\right) \\
& \times \cos \left[q V_{\mathrm{q}}(R) t\right],
\end{aligned}
$$

where $V_{q}(R)$ is the component of $\mathbf{V}(R)$ along $\mathbf{q}$, and $P\left(V_{q}(R)\right)$ is the probability distribution function of $V_{\mathrm{q}}(R)$. If the turbulence is isotropic $P\left(V_{\mathrm{q}}(R)\right)$ is independent of $\mathbf{q}$.

If $P\left(V_{\mathrm{q}}(R)\right)$ is assumed to have the scaling form

$$
P\left(V_{\mathrm{q}}(R)\right)=\bar{u}(R)^{-1} Q\left(V_{\mathrm{q}}(R) / \bar{u}(R)\right),
$$

where $\bar{u}(R)$ is a characteristic scaling velocity, which, in general, will depend on the direction of $q$, then Eq. (2.12) becomes

$$
\begin{aligned}
g(t) & =1+\int_{0}^{L} d R h(R) \int_{-\infty}^{\infty} d\left[V_{\mathrm{q}}(R) / \bar{u}(R)\right] Q\left(V_{\mathrm{q}}(R) / \bar{u}(R)\right) \cos \left[q \bar{u}(R) t V_{\mathrm{q}}(R) / \bar{u}(R)\right] \\
& =1+\int_{0}^{L} d R h(R) F(q \bar{u}(R) t) .
\end{aligned}
$$

The function $F(q \bar{u}(R) t)$ is the Fourier cosine transform of the velocity distribution function $Q\left(V_{\mathrm{q}}(R) / \bar{u}(R)\right)$.

The Kolmogorov theory of fully developed isotropic turbulence ${ }^{10}$ gives

$$
\left\langle[V(R, t)]^{n}\right\rangle=B_{n}\left\langle[V(R, t)]^{2}\right\rangle^{n / 2}=B_{n}(\varepsilon R)^{n / 3},
$$

where $B_{n}$ are apparatus-independent constants and $V(R, t)$ is the amplitude of $\mathbf{V}(R, t)$. Equation (2.15) applies to the separation $R$ in the inertial range $L_{d} \ll R \ll L_{0}$, where $L_{d}$ is the Kolmogorov dissipation length and $L_{0}$ is the outer scale of turbulence. Using Eq. (2.13), we have

$$
\left\langle[V(R, t)]^{n}\right\rangle=\int_{-\infty}^{\infty} d[V(R) / \bar{u}(R)] Q(V(R) / \bar{u}(R))[V(R)]^{n}=B_{n}[\bar{u}(R)]^{n},
$$


where

$$
B_{n}=\int_{-\infty}^{\infty} d z Q(z) z^{n} .
$$

So according to the Kolmogorov picture the characteristic scaling velocity $\bar{u}(R)$ in Eq. (2.13) is

$$
\left.\bar{u}(R)=\langle[V(R), t)]^{2}\right\rangle^{1 / 2}=(\varepsilon R)^{1 / 3},
$$

where $\varepsilon$ is the energy dissipation rate of turbulent flow.

Taking the intermittency of turbulence into account, the $\beta$-model predicts ${ }^{2}$ that

$$
\left\langle[V(R, t)]^{n}\right\rangle=B_{n}(\varepsilon R)^{n / 3}\left(R / L_{0}\right)^{\gamma(3-n)},
$$

with $\gamma=(d-d) / 3$, where $d$ is embedding dimension and $d$ is the fractal dimension of the turbulent active region. In this case, Eq. (2.13) becomes

$$
P\left(V_{\mathrm{q}}(R)\right)=\left(R / L_{0}\right)^{3 \gamma}\left[u^{\prime}(R)\right]^{-1} Q\left(V_{\mathrm{q}}(R) / u^{\prime}(R)\right),
$$

where $u^{\prime}(R)=(\varepsilon R)^{1 / 3}\left(L_{0} / R\right)^{\gamma}$, which is the characteristic turbulent eddy velocity with intermittency corrections. Clearly, $\left(R / L_{0}\right)^{3 \gamma}$ is the probability that a pair of points in turbulent fluid separated by a distance $R$ belongs to the same active region (a homogeneous fractal) of turbulent flow, and $Q\left(V_{\mathrm{q}}(R) / u^{\prime}(R)\right) / u^{\prime}(R)$ is the velocity distribution function in the active region. Then Eq. (2.12) becomes

$g(t)=1+\int_{0}^{L} d R h(R)\left(R / L_{0}\right)^{3 \gamma} F\left(q(\varepsilon R)^{1 / 3} t\left(L_{0} / R\right)^{\gamma}\right)$.

Here $h(R)\left(R / L_{0}\right)^{3 \gamma}$ can be thought as a joint probability that a pair of particles separated by a distance $R$ belongs to the same active region of turbulent flow.

It should be noted that $g(t)$ is not assured of having scaling form $g(t)=g(x)$, where $x$ is defined in Eq. (1.1), merely because the characteristic function $F(y)$ in Eq. (2.21) is of this form. However, if $F(y)$ is an exponentially decaying function of its argument, and $h(R)$ in Eq. (2.21) is an algebraic function of $R$, then it is indeed true that $g(t)=g(x)$, in accordance with our observations. We now examine the implication of the assumption that

$$
F\left(q(\varepsilon R)^{1 / 3} t\left(L_{0} / R\right)^{\gamma}\right)=F(y) \sim \exp (-y),
$$

where

$$
y=q(\varepsilon R)^{1 / 3} t\left(L_{0} / R\right)^{\gamma} .
$$

Inserting Eq. (2.22) and Eq. (2.11) into Eq. (2.21) gives

$$
\begin{aligned}
g(x)=1+2\left(L / L_{0}\right)^{3 \gamma} \exp (-x) \sum_{n=0}^{\infty} x^{n}\{\Gamma((3+9 \gamma) /(1-3 \gamma)+1) /[(1+3 \gamma) \Gamma((3+9 \gamma) /(1-3 \gamma)+1+n)] \\
-\Gamma((6+9 \gamma) /(1-3 \gamma)+1) /[(2+3 \gamma) \Gamma((6+9 \gamma) /(1-3 \gamma)+1+n)]\}
\end{aligned}
$$

Here $x$ is defined in Eq. (1.1) with $\bar{u}(L)=(\varepsilon L)^{1 / 3}$, and $\Gamma(z)$ is the $\gamma$ function. If $d=d(\gamma=0)$, Eq. (2.23) becomes

$g(x)=1+\exp (-x) \sum_{n=0}^{\infty} x^{n}[12 /(n+3) !-720 /(6+n) !]$,

where $x=q \bar{u}(L) t$. Note that the assumption of a single exponential decay of $F(y)$ implies that $P(V(R) / \bar{u}(R))$ is a Lorentzian function. Of course, $P(V(R) / \bar{u}(R))$ cannot remain Lorentzian for very large velocity fluctuations, otherwise its moments higher than the first would diverge.

The above calculation of the correlation function $g(t)$ is made by assuming that the photodetector is an ideal one (the detecting area of the photodetector is infinitesimal small), and that $g(t)$ varies on only one dominant time scale. Of course, the validation of these assumptions must be verified in the real experimental situation.

There are three characteristic times associated with the decay of $g(t)$, in addition to the time $T=\left[q \bar{u}(L)\left(L_{0} /\right.\right.$ $\left.L)^{\gamma}\right]^{-1}$ [see Eq. (1.1)], which characterizes the velocity fluctuations $V(R, t)$. These are the following: (1) the turbulent turnover time, $t_{R}=R / \bar{u}(R)$, associated with eddies of size $R,(2)$ the time associated with the Brownian motion of a diffusing particle $t_{D}=\left(2 q^{2} D\right)^{-1}$, where $D$ is the Stokes diffusion constant, and (3) the particle transit time $t_{U}=\mathscr{D} / U$, where $\mathcal{D}$ is the diameter of the laser beam. Homodyne light scattering spectroscopy is practical as a means of probing the spatial velocity fluctuations in a turbulent flow, only when the decay time $T$ is much less than all three of the above characteristic times, i.e., when

$$
T \ll\left(t_{R^{\prime}} t_{D^{\prime}} t_{U}\right)
$$

From the correlation function $g(t)$ (glance ahead to Fig. 5), one gets a feeling for the typical decay time $T$; it was always less than $100 \mu \mathrm{sec}$ in our experiments. This characteristic time is much shorter than $t_{R}$, which we estimated to be $10^{-3} \mathrm{sec}$ or more. Thus in calculating $g(t)$ it may be assumed that each pair of seed particles separated by a distance $R$, is moving at constant velocity $V(R, t)$ in the time intervals $t$ of interest. This approximation is invoked in relating the measured correlation function to the velocity distribution function $P(\mathbf{V}(R))$. At $q=2.42 \times 10^{5} \mathrm{~cm}^{-1}$, corresponding to a scattering angle of $90^{\circ}, t_{D} \sim 10^{-4} \mathrm{sec}$. Only in the laminar flow domain did diffusion compete with the flow fluctuations in limiting the decay time of $g(t)$. The lifetime broadening effect due to the finite transit time $t_{U}$ was very small in our experiments, the measured decay time $T$ being al- 
ways at least a factor of 10 shorter than $T_{U}$. Thus the condition of Eq. (2.25) is almost always satisfied in our experiments, and the homodyne scattering experiment will almost always be dominated by the contributions of the turbulent fluctuations in the form of Eq. (1.1).

The correlation function measured by the correlator is not properly normalized and has the form

$$
g(t)=\langle n\rangle^{2}[1+H(t)],
$$

where $\langle n\rangle$ is the average number of photon counts per sampling time. The fact that the detecting area of the photomultiplier is of finite size may affect the functional form of $H(t)$. In the simple case where scatterers move independently, as in diffusion, the measured homodyne correlation function $g(t)$ can be factored into parts that depend on the spatial variables (for example, the angle subtended by the photodetector) and the dynamics. ${ }^{20}$ In this case,

$$
g(t)=\langle n\rangle^{2}[1+f(A) G(t)],
$$

where $f(A)$ is a measure of the spatial coherence of the scattered light across the face of the photodetector. When the particles move coherently, as in laminar flow, for example, no such factorization is possible. ${ }^{21,22}$ Nevertheless, in our experiments it was found that for different values of $L, q$, and for large enough $R$, plots of $\log _{10}(H(t))$ versus $\log _{10}(t)$ could be brought into registry by translating the graphs relative to each other both horizontally and vertically. This implies that a homogeneous function can be factored out from $H(t)$ for each $\mathscr{R}$, hence we write $H(t)$ as

$$
H(t)=f(L) G\left(L^{\zeta} q^{\mu} t\right),
$$

where $G$ is a homogeneous function of $L^{\zeta} q^{\mu} t$, and $f(L)$ is the vertical translation factor [analogous to $f(A)$ in Eq. (2.27)]. The function $G$ extracted from our measurements contains information about the dynamics of turbulent fluctuations, and its time dependence is the same as the calculated correlation function $g(t)-1$. The scaling exponents $\zeta$ and $\mu$ are to be determined from the experiment, to be discussed in Sec. IV.

\section{EXPERIMENTAL DETAILS}

Figure 1 shows the physical arrangement of the experimental setup. The fluid, water, is circulated through a closed system by a pump. The water was seeded with polystyrene spheres of diameter $0.06 \mu \mathrm{m}$, the number density of polystyrene spheres being such that their mean spacing is much larger than their diameter (dilute solution) and much smaller than the smallest eddy size of the turbulent flow $L_{d}$. This assures adequate sampling of the turbulent flow. Since the size of the polystyrene spheres is much smaller than the wavelength of the incident light, the scattering by these particles is nearly isotropic. A section of the pipe of $2.0 \mathrm{in}$. diameter, is made of glass to admit the incident laser beam and observe the scattering. Undesirable velocity fluctuations produced by the pump or by the pipe corners, are damped out by a screen (SC) (aperture size is $2.0 \mathrm{~mm}$ ) on the high-pressure side of the

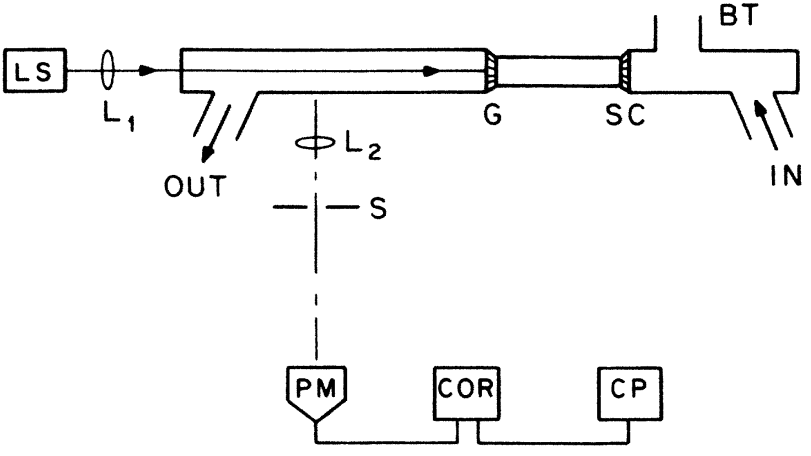

FIG. 1. Schematic diagram of the experimental setup. LS, argon-ion laser; $L_{1}, L_{2}$, lenses; S, slit; G, grid; SC, screen; BT, air bubble trap; PM, photomultiplier; COR, correlator; CP, computer; IN, inlet of flow; OUT, outlet of flow.

grid $(G)$, which generated the turbulence. The aperture size of the grid $M$ was $3.1 \mathrm{~mm}$ and the diameter of the rods with which the grid is made of was $1.5 \mathrm{~mm}$. The measuring point was on the axis of the pipe and $28 \mathrm{~cm}$ downstream from the grid $(y / M=90)$. The circulating fluid was temperature controlled and its mean flow velocity $U$ was varied by changing the pump rotating frequency. Ancillary LDV measurements established that the mean velocity profile was virtually flat rather than parabolic in the direction transverse to that of the mean flow when $R>280$, and that the turbulent intensity $\left\langle\delta v^{2}\right\rangle^{1 / 2} / U$ was $7 \%$ at $R=700$, where $\delta v$ is the fluctuation part of the local velocity. Flow visualization showed that the turbulence in our system did indeed originate from the grid, rather than from other sources.

The lens $L_{1}$ in Fig. 1 focuses the incident beam from an argon-ion laser to make the scattering volume as one dimensional as possible on the axis of the pipe, while $L_{2}$ forms an image of this volume on the slit $S$, of adjustable width $L$. The lens $L_{2}$ is placed in such position that the size of the scattered beam is the same as that of its image (the magnification is 1). It is the light passing through the slit which illuminates the photomultiplier (PM). The water inlet and outlet as well as a stand pipe (BT) where air bubbles can leave the fluid, are shown in Fig. 1. The output pulse train from the photodetector went to a commercial correlator (COR), whose output is $g(t)$. Also indicated is the computer (CP) for storing and analyzing the data. The laser beam was sometimes brought into the fluid along the flow axis, as shown in Fig. 1, but in the $q$ dependence measurements, where the direction of $q$ was kept in parallel with the mean flow direction, it entered through the cylindrical wall of the glass pipe.

\section{RESULTS}

In Sec. II we stated how the measured correlation function was analyzed to establish that $G(t)$ is a scaling function of the control parameters $U, q$, and $L$ [see Eqs. (2.26) and (2.28)]. Here we detail how the exponents $\zeta$ and $\mu$ were determined. When $G(t)$ was plotted versus time $t$ on a $\log -\log$ scale, for fixed $\mathcal{R}$ and $q$ but different 
values of $L$, these curves could be brought into coincidence by sliding one curve horizontally with respect to an arbitrarily chosen one by an amount $\delta(L)$. A plot of $\log [\delta(L)]$ versus $\log (L)$ showed the data points to lie on a straight line, and the slope of the straight line $\zeta$ turned out to be a function of $\mathscr{R}$.

Similarly, it was found that for fixed $\mathscr{R}$ and $L$, but varying $q$, plots of $\log [G(t)]$ versus $\log (t)$ could also be superimposed by translating them horizontally by an amount $\delta(q)$. As long as $\mathscr{R}$ exceeded a certain value $\left(R_{c} \sim 460\right), \delta(q)$ was found to be roughly proportional to $q$. This result is in notable contrast to diffusion, where fluctuations relax at a rate proportional to $q^{2}$. The fact that the decay rate of $G(t)$ is proportional to $q$ rather than $q^{2}$, assures that $\mathbf{V}(R, t)$ is not Gaussianly distributed and that one is not dealing with a diffusive phenomenon.

In the manner described above, it was established that $G(t)=G(x)$ when $R>R_{c}$, where $x$ is proportional to qt $L^{\zeta(R)}$. Since $x$ must be dimensionless, we write it as in Eq. (1.1) with $\bar{u}(L)=(\varepsilon L)^{1 / 3}$, so that $\zeta=\frac{1}{3}-\gamma$. The parameter $\varepsilon$, which has the dimension of the energy dissipation rate in the Kolmogorov theory, is not directly measured in this experiment, nor are any assumptions made about it. Nevertheless, we do find that the velocity $\bar{u}(L)$ which brings our data into the scaling form, is roughly proportional to the mean flow speed $U$. If we use the equation which relates the dissipation rate $\varepsilon$ with the distance behind the grid in the fully developed turbulence case $^{17}\left(\varepsilon \sim U^{3} / M\right)$, our result of $\bar{u}(L) \sim U$ could be thought as an indirect check of $\bar{u}(L) \sim \epsilon^{1 / 3}$.

The scaling behavior of $G(t)$ discussed above is displayed in Fig. 2, which shows $G(x)$ as a function of $x$ for various values of $\theta$ (or $q$ ), $L$, and $\mathcal{R}$. The exponent $\zeta(R)$ could be determined by an alternative method described below. From the scaling behavior of $G(t)$ it is expected that the half-decay time $T$ of $G(t)$ should be proportional to $\left[L^{\zeta} q^{\mu}\right]^{-1}$ [see Eq. (2.28)]. Figure 3 shows

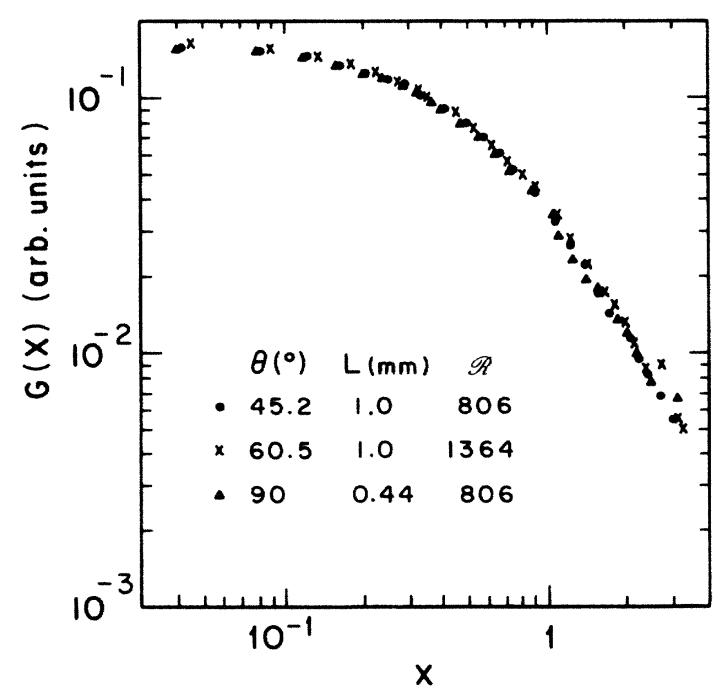

FIG. 2. The scaling function $G(x)=\left\{\left[g(t) /\langle n\rangle^{2}\right]-1\right\} /$ $f(L)$ vs $x=q \bar{u}(L) t\left(L_{0} / L\right)^{\gamma}$ at indicated parameters.

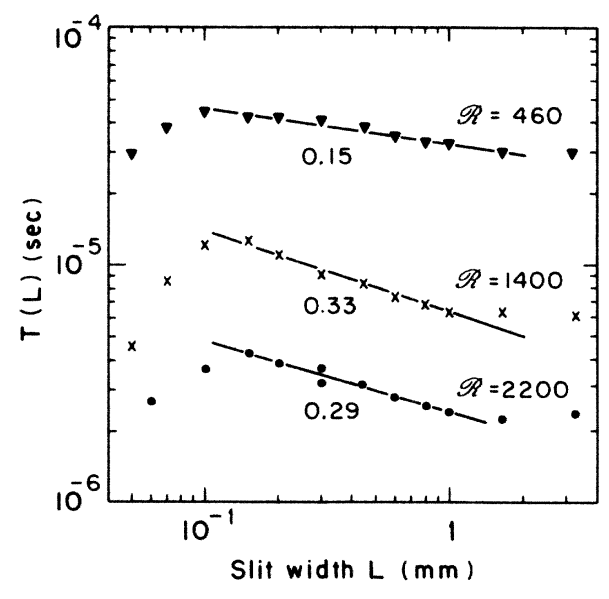

FIG. 3. The half-decay time $T$ of $g(t)$ vs slit width $L$ at $q=2.42 \times 10^{5} \mathrm{~cm}^{-1}$. The number labeled below a line is the slope of that line.

how $T$ varies with slit width $L$ (in $\mathrm{mm}$ ) at a fixed $q=2.42 \times 10^{5} \mathrm{~cm}^{-1}\left(\theta=90^{\circ}\right)$. The three curves correspond to $R=460,1400$, and 2200 . It is seen that increasing $L$ reduces $T$ when $L$ is in the range of 0.1 $\mathrm{mm} \leq L \leq 1.0 \mathrm{~mm}$. This is because opening up the slit increases the size of eddies seen by the photodetector, and larger eddies should have a shorter decay time. The starting point of the power-law behavior of $T$ is at $L=1.0 \mathrm{~mm}$. This limitation was due to the coherence length of the optical system. ${ }^{20}$

Ideally, $T$ should be independent of $L$ when $L<L_{d}$, since there are no eddies smaller than $L_{d}$, and in fact one might hope to measure $L_{d}\left[L_{d} \sim 0.01 \mathrm{~mm}\right.$ at $\left.R=3000\right]$ by finding the value of $L$ at which $T$ levels off as $L$ is decreased. Unfortunately, $L_{d}$ could not be determined in our experiment because the diameter $\mathscr{D}$ of the laser beam could not be reduced below $0.1 \mathrm{~mm}$, and $D L_{d}$. Thus the turnover in $T(L)$ at small $L$ was controlled by the laser beam diameter. In fact, Fig. 3 reveals a decrease in $T$ as $L$ is reduced below the beam diameter, an effect which we do not fully understand. Focusing attention on the decade of $L$, over which $\log (T)$ varies linearly with $\log (L)$ (see Fig. 3), we extract the slope $\zeta$ in the equation, $T \sim L^{-\zeta}$. It is seen that $\zeta$ does not vary with $R$ in a monotonic fashion. The numbers below each straightline segment in Fig. 3 are the values of $\xi$ at the three indicated Reynolds numbers.

Figure 4 shows more clearly the variation of $\zeta$ with $R$ (dashed curve, scale on right). Note the abrupt change of $\zeta$ at $R_{c} \sim 460$. This same kink is also seen in the variation of $T$ itself with $\mathscr{R}$ (solid curve in Fig. 4, scale on left). The latter measurements were made at $q=2.42 \times 10^{5}$ $\mathrm{cm}^{-1}\left(\theta=90^{\circ}\right), L=1.0 \mathrm{~mm}$, and the incident laser beam entered the turbulent stream antiparallel to the flow direction. Somewhat below $\mathcal{R}_{c}$ the profile of the mean velocity, as determined by $\mathrm{LDV}$, has become flat in the direction transverse to the pipe. The flow visualization confirmed that when $R$ is below $\mathcal{R}_{c}$, the flow is already pluglike, a phenomenon one often associates with a chaotic state, and that the flow approaches a homogene- 


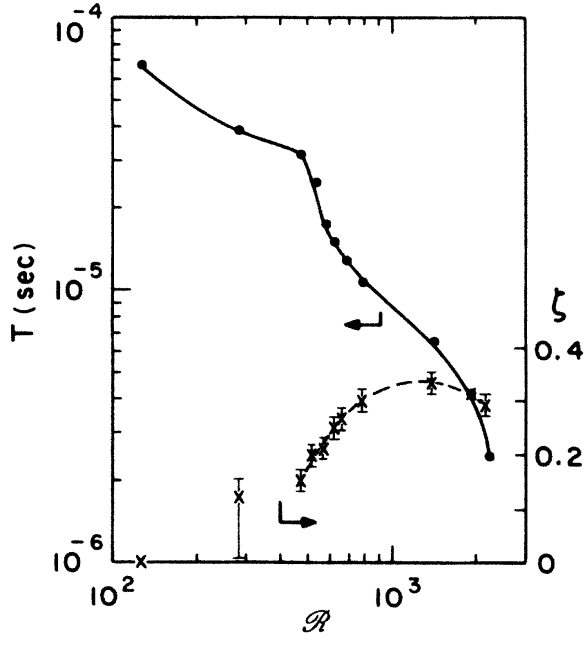

FIG. 4. The variations of the exponent $\zeta(\times)$ (extracted from half-decay time $T$ ) and $T(O)$ with $R$. The solid and dashed curves are drawn by eye through the data points.

ous turbulent state when $\mathcal{R}$ reaches $\mathcal{R}_{c}$. Combining the discussions on Fig. 2, we therefore associate the abrupt change at $R_{c}(\sim 460)$, with the onset of the relative velocity fluctuations being self-similar. As the Reynolds number is increased to above 1400 , the exponent $\zeta$ rises to about $\frac{1}{3}$ which is the Kolmogorov value.

Also measured was the $q$ dependence of the half-decay time $T$. Here the direction of $q$ was kept fixed, namely parallel to the flow direction, while its magnitude was varied. The slit width was also fixed at $L=1.0 \mathrm{~mm}$. To satisfy these conditions it is necessary to vary both the direction of the incident beam and that of the observation, which limited the range of $q$-values that could be spanned. It was found that $T(q)$ also was of power-law form $T \sim q^{-\mu}$. Our measurements of $\mu$ versus $R$ are summarized in Table $\mathrm{I}$, where $\mu$ is given for seven values of $\mathcal{R}$, from 0 to 1860 . Note that when the flow is absent or laminar, the decay time of $g(t)$ is limited by the diffusive motion of seed particles. In this case $T$ $=t_{D}=\left(2 q^{2} D\right)^{-1}$. As the turbulent level is increased, the exponent $\mu$ falls below 1.0, and again increases toward 1.0 , a value which was obtained at $R=1860$. Thus $\mu=1$ is chosen for the scaling form of $G(x)$, where $x$ is defined in Eq. (1.1).

TABLE I. The variation of the exponent $\mu$ with Reynolds number $R$ at $L=1.0 \mathrm{~mm}$. Note: $R=0$ is Brownian motion, $\mathscr{R}=125$ is laminar flow.

\begin{tabular}{lr}
\hline$\mu$ & \multicolumn{1}{c}{$R$} \\
\hline 2.0 & 0 \\
1.92 & 125 \\
0.92 & 280 \\
0.92 & 460 \\
0.76 & 775 \\
0.84 & 1400 \\
1.0 & 1860 \\
\hline \hline
\end{tabular}

In Sec. II it was shown that if the characteristic function $F$ (the Fourier cosine transform of the probability distribution function $P$ ) is an exponentially decaying function of the single variable $y$ in Eq. (2.22), then $g(t)$ has the scaling form $g(x)$ given by Eq. (2.24). This equation was extremely well fitted to our measurements of $g(t)$ in the range of $R \leqslant 1400$ (i.e., when $\gamma \sim 0$ ). An example of this good fit is seen in Fig. 5 (solid line), which shows $g(t)$ at $R=1395, q=2.42 \times 10^{5} \mathrm{~cm}^{-1}$, and $L=0.6$ $\mathrm{mm}$. But what surprised us is that, even when the Reynolds number is in the transition region $(460 \leqslant R \leqslant 1000)$, our data can still be fitted to the same functional form as that in Eq. (2.24). To obtain this fit it was necessary to redefine $x$ as that in Eq. (1.1) and let $\gamma$ be a function of Reynolds number $R$. There is only one adjustable parameter, $T^{\prime}\left(x=t / T^{\prime}\right)$, in this fitting procedure, and $T^{\prime}$ exhibits the same $L$-dependence as that of the half-decay time $T$ as shown in Fig. $3 .^{23}$

The above fitting suggests that the velocity distribution function $P(V(R))$ has the scaling form $P(V(R) / \bar{u}(R))$ even though the flow falls far short of being fully developed. The fitting also indicates that the functional form of this scaling function $P(V(R) / \bar{u}(R))$ is Lorentzian, at least for the most probable part of $P(V(R) / \bar{u}(R))$ since $g(t)$ is relatively insensitive to the rare fluctuations of velocity in our experiment. We note parenthetically that our measurements of $P(V(R))$ are consistent with those obtained in strongly turbulent systems by Anselmet et al. ${ }^{15}$ They find that $P(V(R) / \bar{u}(R))$ decays exponentially over five decades $\left(P\right.$ varies from $10^{-2}$ to $\left.10^{-7}\right)$ when $V(R) / \bar{u}(R)>2$.0. However, the most probable part of $P(V(R) / \bar{u}(R))(P>0.1)$ is clearly not of exponential form, and indeed decays roughly as a Lorentzian function out to $V(R) \simeq 2 \bar{u}(R)$, where $\bar{u}(R)=\left\langle V(R)^{2}\right\rangle^{1 / 2}$.

The finite values of $\gamma$ which we measured as a function of $\mathcal{R}$ and the above fitting assure that our observations cannot be fully explained by either the Kolmogorov theory (in our notation, this theory gives $\gamma=0$ ), or the intermittent turbulence theories, such as the $\beta$-model [where the exponent $\gamma$ is a constant independent upon $R$ ]. The inapplicability of these models is hardly surpris-

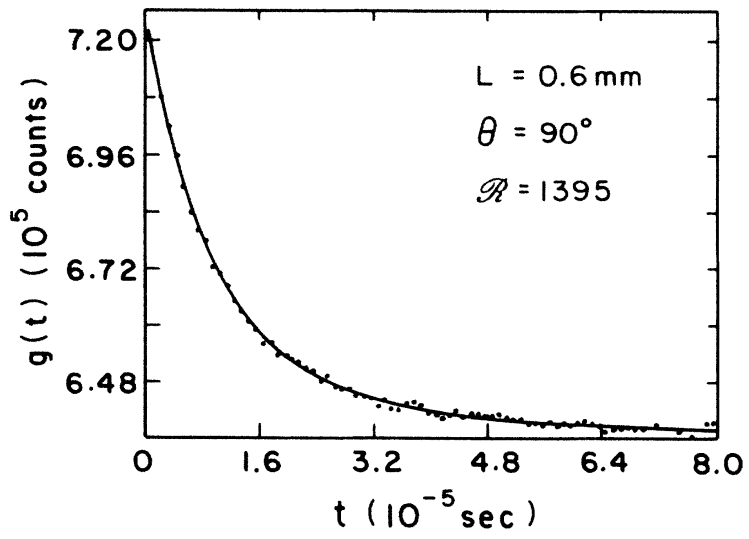

FIG. 5. A typical correlation function $g(t)$ vs $t$. The solid curve is a fit to the incomplete $\gamma$ function in Eq. (2.24). 


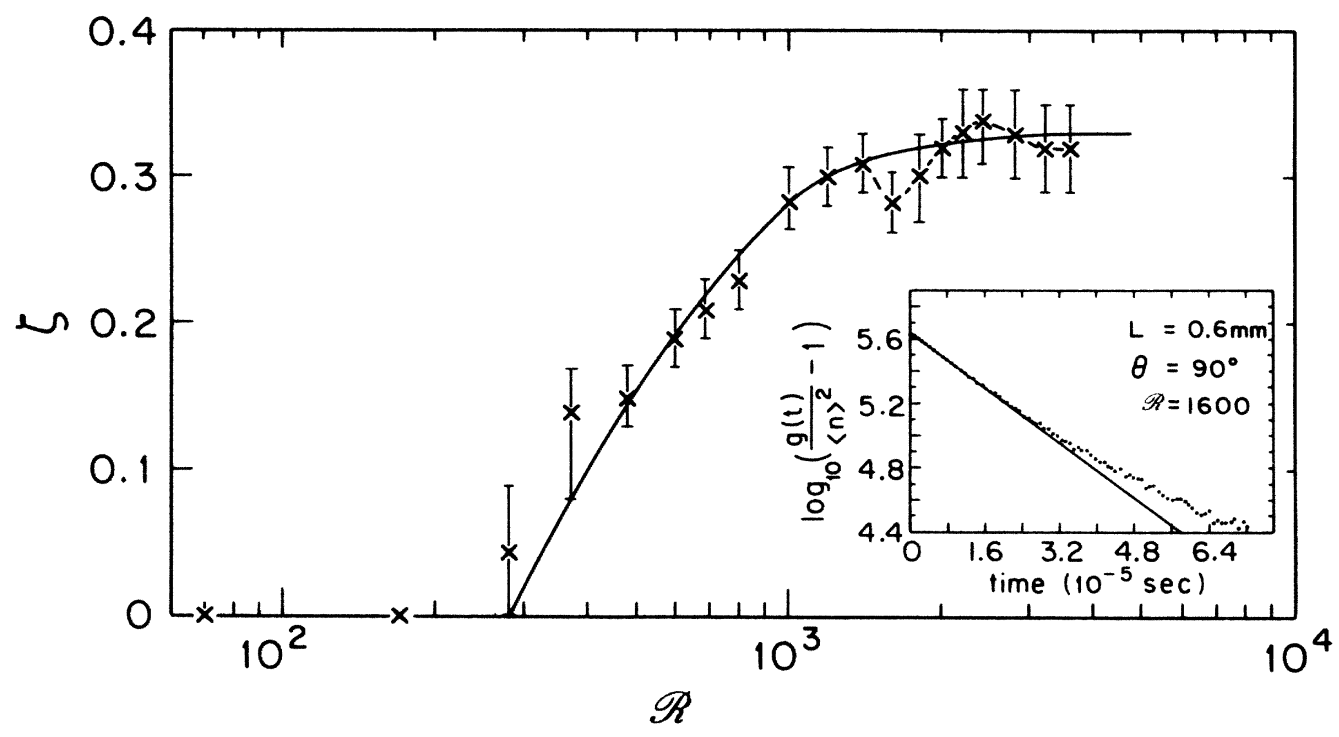

FIG. 6. The variation of the exponent $\zeta$ (extracted from $\Gamma$ ) with $\mathcal{R}$. The solid curve is drawn by eye through the data points, and the dashed curve shows the oscillatory behavior of $\zeta$. The inset is a plot of $\log _{10}\left[g(t) /\langle n\rangle^{2}-1\right]$ vs $t$ at indicated parameters.

ing, considering that only moderate Reynolds numbers are reached in our experiments. What is pleasingly unexpected is that the flow does exhibit a striking self-similar behavior as long as $R$ exceeded $\mathcal{R}_{c}$.

It is also known from Eq. (2.24) that for small values of $t, \log _{10}[g(t)-1]$ is a linear function of time $t$ with a slope proportional to $q \bar{u}(L)$. The inset in Fig. 6 shows a plot of $\log _{10}\left[g(t) /\langle n\rangle^{2}-1\right]$ versus time $t$ at $\mathcal{R}=1600$, $\theta=90^{\circ}$, and $L=0.6 \mathrm{~mm}$. It can be seen that only at large $t$ does the curve start to deviate from linear behavior. The reciprocal of the slope of the straight line fitted from short-time data gives a typical relaxation time $\Gamma$ of $g(t)$. With this motivation, we analyzed another set of correlation functions measured with various values of $R, L$ and at fixed $q\left(\theta=90^{\circ}\right)$. A semilog plot of $g(t) /\langle n\rangle^{2}-1$ versus $t$ was made for each $g(t)$ to extract the relaxation time $\Gamma$. For fixed $R$ and $q$ but different values of $L, \Gamma$ as a function of slit width $L$ obeys a power law, $\Gamma(L) \sim L^{-\zeta}$ (a log-log plot of $\Gamma$ versus $L$ is very similar to that in Fig. 3 ), and the exponent $\zeta$ turns out to be a function of $\mathcal{R}$.

The variation of $\xi$ with $R$ determined in the way described above is displayed in Fig. 6. It is clear that Fig. 6 and Fig. 4 are similar for the same covered region of $\mathcal{R}$; but at the higher $R$ end, Fig. 6 shows more clearly that $\zeta(\mathcal{R})$ saturates at a value close to $\frac{1}{3}$ (Kolmogorov value). In addition, it can be seen in Fig. 6 that there is a small amplitude oscillation superimposed on the saturation line of $\zeta$, while Fig. 4 only shows the onset of the oscillation. This oscillatory behavior of $\zeta$ was also seen at the same region of mean flow velocities when the grid was removed. It should be noted that the amplitude of these oscillations is comparable to the experimental uncertainty in $\zeta$.

It was found that when $g(t) /\langle n\rangle^{2}-1$ was plotted versus $t$ on a semilog scale, for fixed $L$ and $q$, the deviation of these curves from a straight line gets larger as $\mathscr{R}$ is increased (i.e., the number of data points lying on a straight line decrease). This kind behavior was also observed from curves of $\log _{10}\left[g(t) /\langle n\rangle^{2}-1\right]$ versus $t$ at fixed $R$ and $q$ but increasing values of $L$. The above observations can be understood, at least qualitatively, from the energy cascade picture of turbulence. Our calculation of the correlation function shows that $g(t)$ is basically a weighted summation over many exponentials involving various length scales. As $\mathscr{R}$ is increased, the turbulent energy cascades down through an increasing number of length scales. Since eddies at each length scale add another exponential to the correlation function, the deviation of $g(t)$ from pure exponential behavior should increase as $\mathcal{R}$ (or $L$ ) is increased.

To further study the transition at $\mathcal{R}_{c} \sim 460$ in Fig. 4, the exponent $\zeta$ as a function of $\left[R-\mathscr{R}_{c}\right] / \mathscr{R}_{c}$ is plotted on a $\log -\log$ scale in the vicinity of $\mathcal{R}_{c}$. It was found that all the data points lie on a straight line over about one and a half decades. So we have

$$
\zeta(\mathscr{R}) \sim\left\{\left[\mathcal{R}-\mathscr{R}_{c}\right] / \mathscr{R}_{c}\right\}^{\alpha},
$$

with $\alpha=0.21 \pm 0.07$. If $R_{c}$ is taken as 280 , as in Fig. 6, $\alpha=0.45 \pm 0.08$.

\section{SUMMARY}

We have studied grid-generated turbulence with the rarely exploited technique of photon correlation spectroscopy. Measurements of this type give access to the probability distribution function $P(V(R))$ that a pair of particles in the fluid, having separation $R$, differ in velocity by $V(R, t)$. At moderate Reynolds number the intensity correlation function $g(t)$ has the scaling form $g(x)$, where $x=q \bar{u}(L) t\left(L_{0} / L\right)^{\gamma}$, and $\bar{u}(L)=(\varepsilon L)^{1 / 3}$ is the characteristic eddy velocity. This self-similar behavior is seen only when $\mathscr{R}_{>} \mathscr{R}_{c}\left(\mathcal{R}_{c} \sim 460\right)$. All measurements 
ments suggest that the flow changes its character at this point. The exponent $\zeta$ (or $\gamma$ ) may be regarded as an additional parameter that is needed to characterize the flow at moderate turbulent levels. In the vicinity of $\mathcal{R}_{c}, \zeta$ as a function of $R$ is approximately of the form $\zeta \sim\left[\left(\mathcal{R}-\mathcal{R}_{c}\right) / \mathcal{R}_{c}\right]^{\alpha}$. Our measurements are consistent with the assumption that $P(V(R) / \bar{u}(R))$ is of Lorentzian form at small values of $V(R, t)$. Equivalently the characteristic function $F(y)$ decays exponentially.

\section{ACKNOWLEDGMENTS}

The authors of this paper are indebted to M. Nelkin and J. Stavans for stimulating comments and especially to A. Onuki for the insights he has provided. In its early stages, this research received valuable stimuli from $\mathbf{I}$. Procaccia. Our work is supported by the National Science Foundation under Contract No. DMR-8611666.
${ }^{1}$ C. W. Van Atta and J. Park, in Statistical Models and Turbulence, Vol. 12 of Lectures Notes in Physics, edited by M. Rosenblatt and C. W. Van Atta (Springer, Berlin, 1972), p. 402.

${ }^{2}$ U. Frisch, P. Sulem, and M. J. Nelkin, J. Fluid Mech. 87, 719 (1978).

${ }^{3}$ B. Chu, Laser Light Scattering (Academic, New York, 1974).

${ }^{4}$ P. J. Bourke, et al., J. Phys. A 3, 216 (1970).

${ }^{5}$ L. E. Drain, The Laser Doppler Technique (Wiley, New York, 1980).

${ }^{6}$ F. Durst, A. Melling, and J. H. Whitelaw, Principles and Practice of Laser-Doppler Anemometry, 2nd ed. (Academic, New York, 1981).

${ }^{7}$ G. G. Fuller, et al., J. Fluid Mech. 100, 555 (1980).

${ }^{8}$ A. V. Smolyakov and V. M. Tkachenko, The Measurement of Turbulent Fluctuations, (Springer, Berlin, 1983).

${ }^{9}$ J. L. Lumley, Phys. Fluids 8, 1056 (1965).

${ }^{10}$ A. N. Kolmogorov, C. R. (Dokl.) Acad. Sci. USSR, 30, 301 (1941); 31, 538 (1941).

${ }^{11}$ R. H. Kraichnan, J. Fluid Mech. 62, 305 (1974).

${ }^{12} \mathrm{U}$. Frisch, in Turbulence and Predictability in Geophysical Fluid Dynamics and Climate Dynamics, edited by M. Ghil, R. Benzi, and G. Parisi (North-Holland, New York, 1985), p. 71.

${ }^{13}$ H. A. Rose and P. L. Sulem, J. Phys. (Paris) 39, 443 (1978).

${ }^{14}$ J. A. Dutton and D. G. Deaven, Radio Sci. 4, 1341 (1969).
${ }^{15}$ F. Anselmet et al., J. Fluid Mech. 140, 63 (1984).

${ }^{16}$ R. A. Antonia, B. R. Satyprkash, and A. K. Hussain Phys. Fluids 25, 29 (1982).

${ }^{17}$ C. H. Gibson and W. H. Schwarz, J. Fluid Mech. 16, 364 (1963).

${ }^{18}$ H. Jennekes and J. L. Lumley, $A$ First Course in Turbulence (MIT, Cambridge, Mass., 1972), Chap. 8.

${ }^{19}$ In Eq. (2.10) (and hereafter) we have ignored the fact that the integral over $R$ has a lower cut-off $L_{d}$. This approximation is valid when $L_{d} / L \ll 1$, i.e., we have assumed that a small fraction of the integral in Eq. (2.10) comes from the interval $\left[0, L_{d}\right]$.

${ }^{20}$ B. J. Berne and R. Pecora, Dynamic Light Scattering (Wiley, New York, 1976).

${ }^{21}$ In general one cannot factor $g(t)$ into spatial- and timedependent parts, though this factorization is exact if the source of the scattering has a Gaussian distribution.

${ }^{22}$ N. A. Clark, B. J. Ackerson, and T. W. Taylor, J. Phys. (Paris) Colloq., 3, C137 (1985).

${ }^{23}$ In more detail the fitting procedure was as follows: the parameter $T^{\prime}$ was first extracted from fittings. For fixed $R$ and $q$ but different values of $L$, a $\log -\log$ plot of $T^{\prime}$ vs $L$ shows that $T^{\prime}$ roughly obeys a power law, $T^{\prime} \sim L^{-\zeta}$. The exponent $\zeta$ found from the log-log plot turns out to be a function of $\mathcal{R}$. 